\title{
Unsteady and Post-Stall Aerodynamic Modeling for Flight Dynamics Simulation
}

\author{
Ryan Paul* , Joseba Murua $^{\dagger}$, and Ashok Gopalarathnam ${ }^{\ddagger}$
}

\begin{abstract}
This paper presents a methodology for flight dynamics simulation including three-dimensional unsteady and post-stall aerodynamics. This is accomplished by integrating a decambering viscous correction into a linearized unsteady vortex lattice method. Coupling the aerodynamic model with the nonlinear rigid-aircraft equations of motion results in a low-order, medium-fidelity framework for flight dynamics simulation. The numerical studies first evaluate the importance of unsteady aerodynamic effects on the dominant aircraft modes, illustrating the errors incurred on the prediction of the short period when quasi-steady approximations are employed. Next, the combined impact of unsteady and post-stall aerodynamics is assessed on a maneuvering aircraft. Furthermore, the framework is expected to be a suitable design-oriented tool for flight control synthesis and to incorporate aeroelastic modeling.
\end{abstract}

\section{Introduction}

High fidelity aerodynamic models implemented in the form of look-up tables [1-3] are commonplace in certified flight simulators and training devices. Force and moment information for these look-up tables is commonly developed using some combination of the following techniques: Computational Fluid Dynamics (CFD), experimental testing of models in wind tunnels (static and dynamic), and/or experimental flight testing. Both CFD methods and data from experiments are capable of representing forces and moments in the nominal flight regime, where aerodynamics are expected to be linear, and beyond this range where significant amounts of flow separation exist (aerodynamic stall). The primary disadvantage of using these high-fidelity representations of forces and moments is the significant effort required to develop the look-up table. Each expected operating condition, defined not only by the aerodynamic inflow angles but also three components of angular rates, derivatives of these rates, and other explanatory variables as desired, must be run as a CFD or experimental test case to develop the look up table. It is often not feasible to perform such an extensive study due to limited resources, or because the required level of detail is simply not available at early conceptual design stages.

The methodology presented in this research represents a departure from the high-fidelity approach discussed above. A medium fidelity aerodynamics model is considered, based on an unsteady vortex lattice method (UVLM) $[4,5]$ with a post-stall model based on iterative decambering $[6,7]$.

The UVLM is a fully unsteady, three-dimensional aerodynamic method, which is very accurate as long as potentialflow conditions are satisfied [8] - in practice this requires low speed, attached flow. However, with increasing angles of attack, the boundary layer on the upper surface of a wing thickens and finally separates. If separation occurs, then potential-flow predictions deviate from the real viscous flow. The underlying idea of the decambering methodology is that this mismatch due to the boundary-layer displacement thickness and separation can be related to an effective change in the chordwise camber. In other words, the goal is to match the potential-flow solution to the viscous one by introducing a decambering variable, which is effectively a camber correction - it can also be seen as a rotation of the airfoil, modifying the effective angle of incidence to fit viscous data. This idea has been around for several decades, but most recent progress can be found in Refs. [6,9-12]. While the scheme relies on a 2D airfoil data, 3D effects can be incorporated by accounting for the aerodynamic interference among all lifting-surface airfoils. This can be easily done on the UVLM, for instance, when enforcing the boundary conditions. A strip-theory philosophy for engineeringlevel predictions of wing aerodynamics at high angles of attack has been extensively adopted before [13-17], leading

\footnotetext{
${ }^{*}$ Graduate Research Assistant, Department of Mechanical and Aerospace Engineering, North Carolina State University, Box 7910, Raleigh, NC, rcpaul@ncsu.edu. Student Member AIAA

${ }^{\dagger}$ Lecturer in Aerospace Engineering, University of Surrey, UK, j.murua@surrey.ac.uk. Member AIAA

¥Associate Professor, Department of Mechanical and Aerospace Engineering, North Carolina State University, Box 7910, Raleigh, NC, ashok_g@ncsu.edu, (919) 515-5669. Associate Fellow AIAA
} 
to commendable success in modeling post-stall flight dynamics [18]. In fact, the present approach has already been demonstrated to hold very well for unswept planforms [7].

The approach discussed in this manuscript is novel in that it includes an unsteady aerodynamics model and a scheme for the computation of post-stall aerodynamics when appropriate. The resulting model is expected to provide a powerful toolbox for the near-real-time simulation flight dynamics, and should also pave the way for the simulation of the dynamics of flexible aircraft, including aeroelasticity, in the near future.

\section{Unsteady Vortex Lattice Method}

The unsteady vortex lattice method (UVLM) is an efficient computational technique to solve 3-D potential-flow problems about moving (and deforming) lifting surfaces. The basics of the standard UVLM algorithm are described by Katz and Plotkin [4], but an alternative formulation will be used herein, following closely that presented in Ref. [5] whereby the governing equations are written in state-space form, ideally suited for flight simulation.
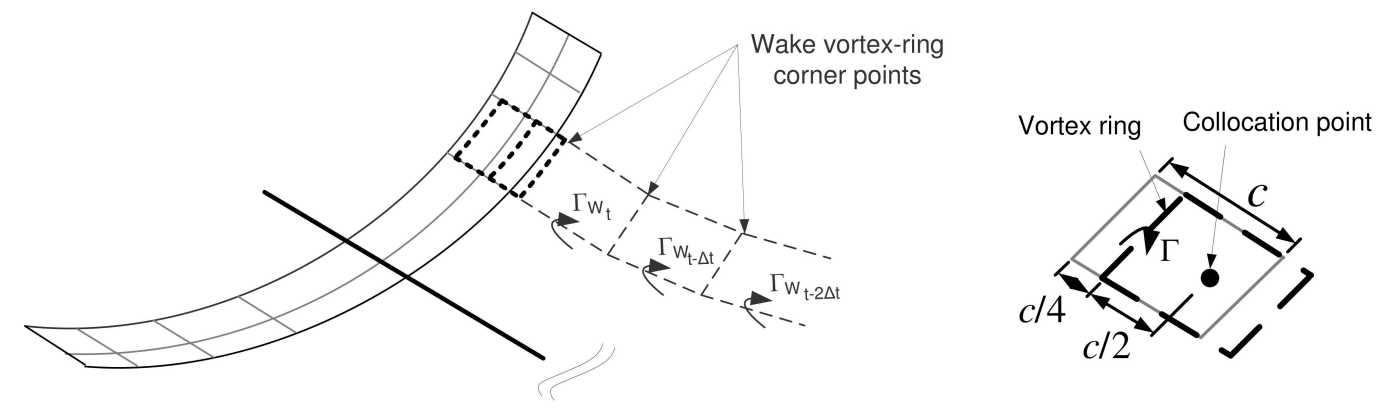

Figure 1: Unsteady aerodynamics model: lifting-surface and wake discretization using vortex-ring elements.

In the UVLM, elementary solutions are distributed over lifting surfaces and the nonpenetration boundary condition is imposed at a number of control (collocation) points. The elementary solution is the so-called vortex ring, distributed over the instantaneous mean surface, ignoring thickness. The leading segment of the vortex ring lies on the panel's quarter-chord line, and a collocation point is placed at the three-quarter-chord line, which falls at the center of the vortex ring (see Figure 1). As the surface moves along its flight path, a force-free wake is obtained as part of the solution procedure, also represented by vortex rings. By imposing the non-penetration boundary condition at a number of collocation points, a system of algebraic equations is obtained. Thus, at discrete time step $n+1$, the vorticity distribution of the bound vortex elements is determined by

$$
A_{c b} \boldsymbol{\Gamma}_{b}^{n+1}+A_{c w} \Gamma_{w}^{n+1}+\boldsymbol{w}^{n+1}=0,
$$

where $\boldsymbol{\Gamma}_{b}$ and $\boldsymbol{\Gamma}_{w}$ are the column vectors with the circulation strengths in the bound and wake vortex-rings, respectively; $A_{c b}=A_{c b}\left(\zeta_{b}\right)$ and $A_{c w}=A_{c w}\left(\zeta_{b}, \zeta_{w}\right)$ are the wing-wing and wing-wake aerodynamic influence coefficient matrices, computed at the collocation points through the Biot-Savart law; and $\zeta_{b}$ and $\zeta_{w}$ are the column vectors with the bound and wake grid coordinates. $\boldsymbol{w}$ in Eq. (1) is the column vector of normal components of all velocities except those induced by bound and wake vorticity, which may encompass deployment of control surfaces, gust induced velocities, wing deformations and rigid-body motions, and will be denoted here as "non-circulatory velocity". It also includes the contribution of the decambering, when applicable in the post-stall regime, as explained in Section IV. The time at which the different variables are evaluated within the current time step is determined by $t^{n+\epsilon}=t^{n}+\epsilon \Delta t$, with $0 \leq \epsilon \leq 1$, and depends on the integration scheme.

At each time step, as the circulation of the wing changes, a new row of vortex rings will be shed into the wake from the trailing edge of each lifting surface. In addition to this, the existing wake may be displaced following the local flow velocity (free-wake model). An accurate description of a force-free wake might be essential when it impinges on a body, such as wake encounters on trailing aircraft or blade-vortex interactions on rotorcraft. However, its impact on the fixed-wing-aircraft flight dynamics considered in this work has been shown to be minimal [19]. As a result, and as it is a computationally very expensive process that requires the evaluation of the local velocities at the wake grid-points, a fixed wake model was used for the current work. The propagation equations for the wake circulation can be written in discrete time as

$$
\boldsymbol{\Gamma}_{w}^{n+1}=C_{\Gamma b} \boldsymbol{\Gamma}_{b}^{n}+C_{\Gamma w} \boldsymbol{\Gamma}_{w}^{n}
$$


where $C_{\Gamma b}$ and $C_{\Gamma w}$ map the circulation of the previous time step to the current one, and they are very sparse constant matrices which account for Kelvin's circulation theorem and Helmholtz's vortex theorem.

Once the distribution of vorticity has been obtained at each time step, the inviscid aerodynamic loads can be computed. Different methods exist, such as the expressions given by Katz and Plotkin [4], which are based on the unsteady Bernoulli equation but use the small-angle assumption and introduce an approximation for induced drag in order to account for leading edge suction. The method used in the current work is the Joukowski theorem applied at each bound vortex segment midpoint. While the Joukowski theorem is more computationally expensive than the unsteady Bernoulli [20], it provides a more general approach and avoids the small-angle assumption, deemed more appropriate for the high angle of attack work. The aerodynamic forces are given by

$$
\overrightarrow{F_{i n v}}=\rho \overrightarrow{V_{l c}} \times \overrightarrow{\Gamma_{b}}
$$

where $\vec{V}_{l c}$ is the total velocity (kinematic + induced) at the point of lift-calculation. For a given strip, this velocity is calculated as

$$
\vec{V}_{l c}=-\left[\begin{array}{c}
u \\
v \\
w
\end{array}\right]-\left[\begin{array}{c}
p \\
q \\
r
\end{array}\right] \times \vec{r}_{l c}+\left[\begin{array}{c}
H_{l b_{x}} \boldsymbol{\Gamma}_{b} \\
H_{l b_{y}} \boldsymbol{\Gamma}_{b} \\
H_{l b_{z}} \boldsymbol{\Gamma}_{b}
\end{array}\right]+\left[\begin{array}{c}
H_{l w_{x}} \boldsymbol{\Gamma}_{w} \\
H_{l w_{y}} \boldsymbol{\Gamma}_{w} \\
H_{l w_{z}} \boldsymbol{\Gamma}_{w}
\end{array}\right]
$$

where

$$
\vec{r}_{l c}=\left[\begin{array}{lll}
x_{l c}-x_{C G} & y_{l c}-y_{C G} & z_{l c}-z_{C G}
\end{array}\right]
$$

The $H_{l b}$ and $H_{l w}$ matrices [21] are similar to aerodynamic influence coefficient matrices, except that they contain induced velocity information computed at the lift calculation points, $\vec{r}_{l c}$ by the bound and wake vortex rings, respectively. Three $H_{l b}$ and $H_{l w}$ matrices are needed to store the induced velocity components projected along the body-axis $x, y$, and $z$ directions by a unit strength vortex ring.

The above equations correspond to the general nonlinear formulation of the UVLM, where the nonlinearities arise due to the enforcement of the boundary conditions on the deformed geometry and the free-wake model, and hence can account for large motions of the lifting surfaces in unsteady flow. The number of operations at each time step in the UVLM is of the order of $O\left(N^{2}\right)$, where $N$ is the number of total vortex rings, including lifting surfaces and wakes, and the size of the latter grows linearly with time. There are a number of ways of reducing this computational burden, such as fast multipole algorithms [22], or less sophisticated but easier to implement solutions, such as prescribed wake assumptions and/or truncation.

The general equations can also be linearized [5], which proves useful for problems such as stability analysis or design of linear control laws. In this context, the linearization will generally take place about a trimmed aircraft configuration and is obtained through small perturbation analysis in body-fixed axes. In addition, a frozen aerodynamic geometry is assumed (both in the lifting surfaces and wakes), which effectively implies that the aerodynamic influence coefficient matrices $A_{c b}$ and $A_{c w}$ are computed only once and remain constant. $H_{l b}$ and $H_{l w}$ also remain constant under this assumption. This linearization assuming a frozen aerodynamic geometry leads to a descriptor state-space form of the linear UVLM as [23]

$$
\begin{aligned}
E_{A} \Delta \boldsymbol{x}_{A}^{n+1}+F_{A} \Delta \boldsymbol{u}_{A}^{n+1} & =A_{A} \Delta \boldsymbol{x}_{A}^{n}+B_{A} \Delta \boldsymbol{u}_{A}^{n}, \\
\Delta \boldsymbol{y}_{A}^{n} & =C_{A} \Delta \boldsymbol{x}_{A}^{n}+D_{A} \Delta \boldsymbol{u}_{A}^{n},
\end{aligned}
$$

where the outputs $\boldsymbol{y}_{A}$ are the aerodynamic loads, and the states and inputs that fully define the linearized aerodynamic system are

$$
\boldsymbol{x}_{A}=\left\{\begin{array}{l}
\boldsymbol{\Gamma}_{b} \\
\boldsymbol{\Gamma}_{w} \\
\dot{\boldsymbol{\Gamma}}_{b}
\end{array}\right\}, \quad \text { and } \quad \boldsymbol{u}_{A}=\left\{\begin{array}{c}
\zeta_{b} \\
\dot{\boldsymbol{\zeta}}_{b}
\end{array}\right\} .
$$

\section{Post-Stall Aerodynamics Using Iterative Decambering}

This section discusses the solution procedure for applying decambering to a finite wing at some angle of attack to illustrate the concept and show how it is applicable to the current work. While this discussion focuses on applying decambering to a finite wing, the concepts are extensible to configurations of several lifting surfaces. 
Decambering is straightforward to apply in $2 \mathrm{D}$ aerodynamics. The camber reduction is simply set at the required value to tilt the local section such that the reduction in lift due to viscous effects is matched by the potential flow solution. In a 3D aerodynamics analysis method, application of decambering is most easily thought of as a rotation of the normal vector for all chordwise panels in a spanwise strip by a decambering angle, $\delta_{1}$. However, finding the correct value of $\delta_{1}$ to set for each strip on a 3D wing is not straightforward because the modification of a particular sections $\delta_{1}$ is likely to have an effect on the neighboring strips and on lifting surfaces downstream of this strip. Because of the effect that changing one strips camber has on other sections, the decambering procedure is implemented in a Newton type iteration, which takes the form:

$$
\mathbf{J} \cdot \delta \mathbf{x}=-\mathbf{F},
$$

where $-\mathbf{F}$ is the residual driven to zero through the iterative process. Hence, while the input data relies on $2 \mathrm{D}$ viscous curves, the approach is $3 \mathrm{D}$, since the interference among all strips is accounted for until convergence is reached.

The decambering procedure may be described as follows:

\section{Assume initial decambering}

Begin with an assumed $\delta_{1}$ value for each spanwise section, denoted by $\boldsymbol{\delta}_{\mathbf{1}, \boldsymbol{s}}$. Typically, no decambering is applied initially and $\delta_{1, s}=0$.

\section{Calculate initial operating points}

The potential flow analysis method is used to determine the starting section lift coefficient, $\boldsymbol{C}_{\boldsymbol{l}}$, for each spanwise section using the unsteady vortex lattice method described in Section II. The effective angle of attack for each spanwise section is determined through

$$
\alpha_{e f f}=\left(C_{l}\right)_{s e c} / a_{0}-\delta_{1}-\alpha_{0 l}
$$

Figure 2 shows these initial operating points for the sections on a finite wing for both a low and high angle of attack decambering computation. In the low angle of attack case, we can clearly see that the starting points lie on the input viscous data, as the solution exists in the linear aerodynamic regime. For the high angle of attack case, however, starting solutions for each operating point lie on the potential flow lift curve.
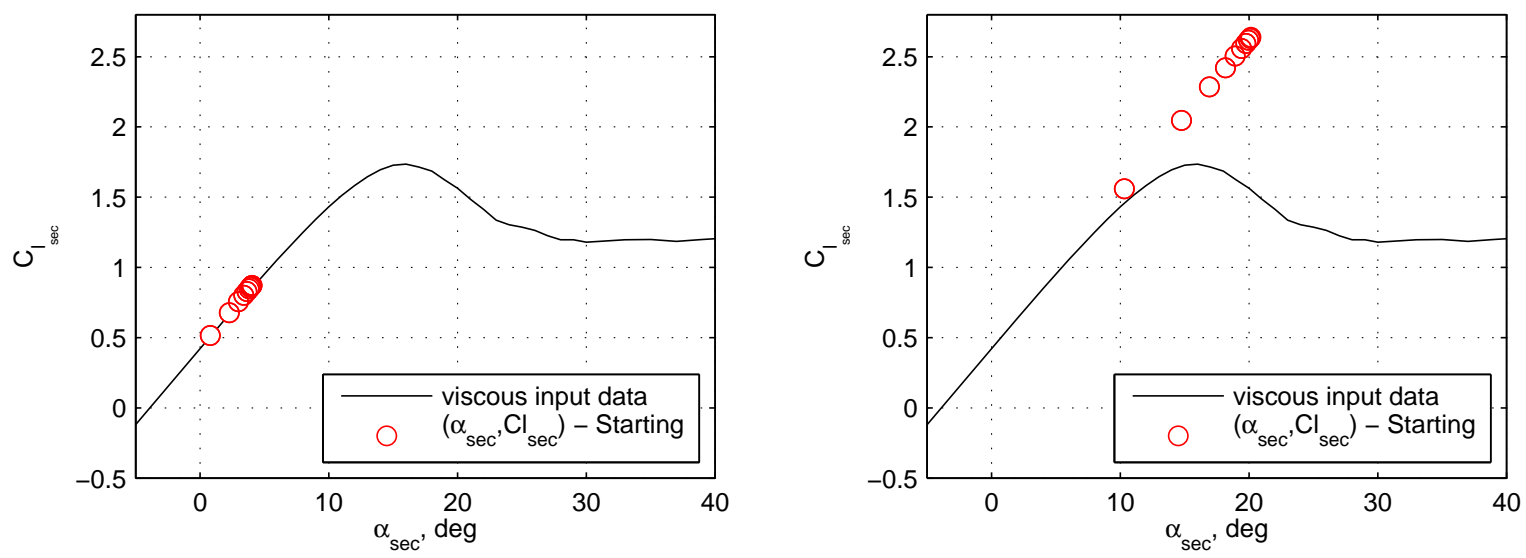

Figure 2: Decambering solution for sections of a rectangular wing initialized with all $\delta_{1}=0$.

\section{Calculate residual}

The residual, from Eq. (7), is calculated for each operating point. In the decambering formulation the residual represents the $\Delta \boldsymbol{C}_{\boldsymbol{l}}$ between a current operating point, $\left(\boldsymbol{\alpha}_{\boldsymbol{s e c}}, \boldsymbol{C}_{\boldsymbol{l}_{\mathbf{s e c}}}\right.$, and some unknown "target" point, $\left(\boldsymbol{\alpha}_{t}, \boldsymbol{C}_{\boldsymbol{l}_{t}}\right)$, which exists on the viscous input data, such that

$$
F=C_{l_{s e c}}-C_{l_{t}}
$$

For each section, the line joining the current operating point and the target point on the viscous curve is called the "trajectory line." Unlike in a typical Newton iteration, where the residual calculation is very straightforward, it is not clear how to project the current section operating points onto the viscous input data to facilitate the 
residual calculation. In other words, there is no clear way to determine the slope of the trajectory lines. The difficulty is that the target point for any section will depend on the unknown decambering at other sections. The current method of residual calculation is an extension of Scheme D in Ref. 24. In this approach, the slope of the trajectory line for any section $i$ is determined by making a small perturbation, $\Delta \delta_{1}(i)$, in the decambering angle, $\delta_{1}(i)$, and determining the resulting change in the operating point, $\left(\alpha_{s e c}(i), C_{l_{s e c}}(i)\right.$, for that section. In determining this slope, it has been assumed that every other section $j$ will also have a perturbation to its $\delta_{1}(j)$ which will be equal to $\Delta \delta_{1}(i)$ multiplied by the cosine of the dihedral-angle difference between sections $i$ and $j$. It has been found that trajectory lines determined in this manner result in the estimated target points $\left(\boldsymbol{\alpha}_{t}, \boldsymbol{C}_{\boldsymbol{l}_{t}}\right)$ being very close to the final converged solution for $\left(\boldsymbol{\alpha}_{t}, \boldsymbol{C}_{\boldsymbol{l}_{t}}\right)$. As a result, the iteration typically converges in approximately 5 iterations with no requirement for under relaxation [24]. Additionally, these trajectory-line slopes are independent of operating condition and decambering and can be precomputed once and reused during the simulation.

4. Modify $\delta_{1}$ vector

After the target point is identified, the $\delta_{1}$ vector is modified to move the current operating points closer to the input $C_{l}-\alpha$ data. The manner in which the $\delta_{1}$ vector is altered is dependent on the residual calculation method.

After initialization, the final two steps of the decambering procedure are repeated until all operating points are brought into a specified tolerance of the input data. Figure 3 shows the starting and converged operating points for a rectangular wing analyzed at a pre and post-stall angle of attack.
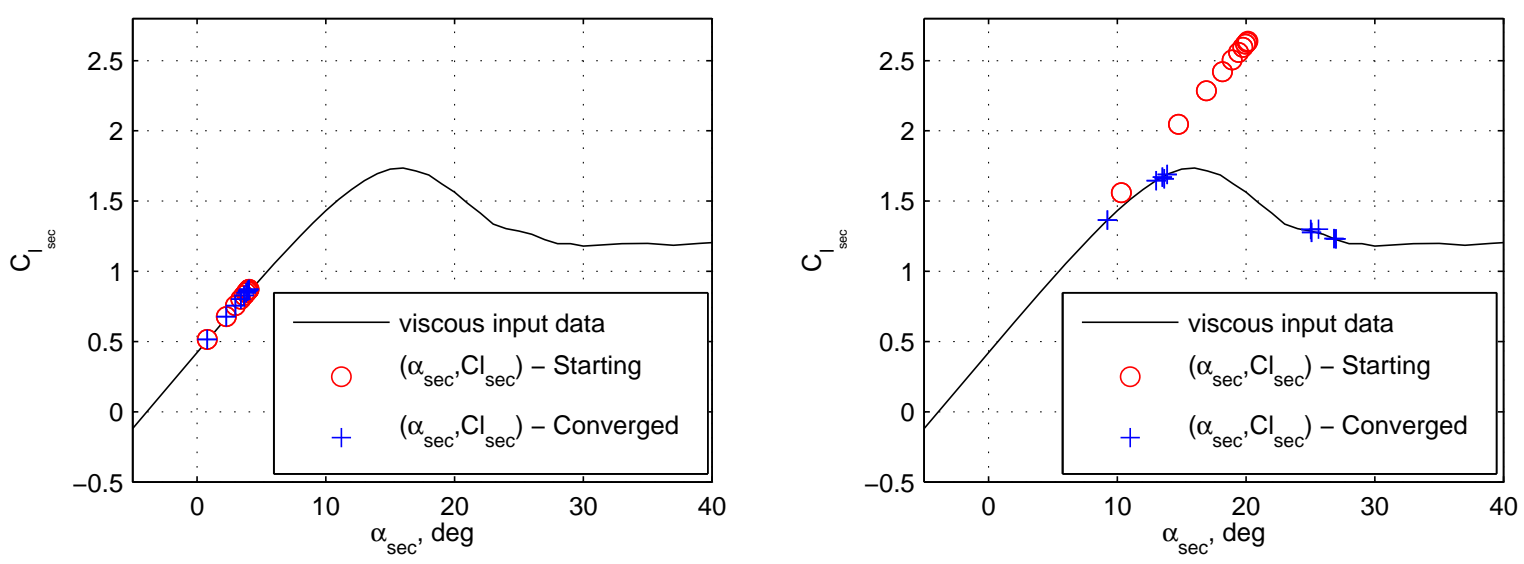

Figure 3: Decambering solution for sections of a rectangular wing showing starting and converged solutions for prestall and post-stall angles of attack.

\section{Simulation of Post-Stall Flight Dynamics}

The objective of the current research is to incorporate the post-stall model of Section III into the unsteady aerodynamics analysis method presented in Section II and integrate the resulting aerodynamic model with the aircraft equations of motion for a flight dynamics simulation framework. The methodology for the combined model is presented as a flowchart in Figure 4. 


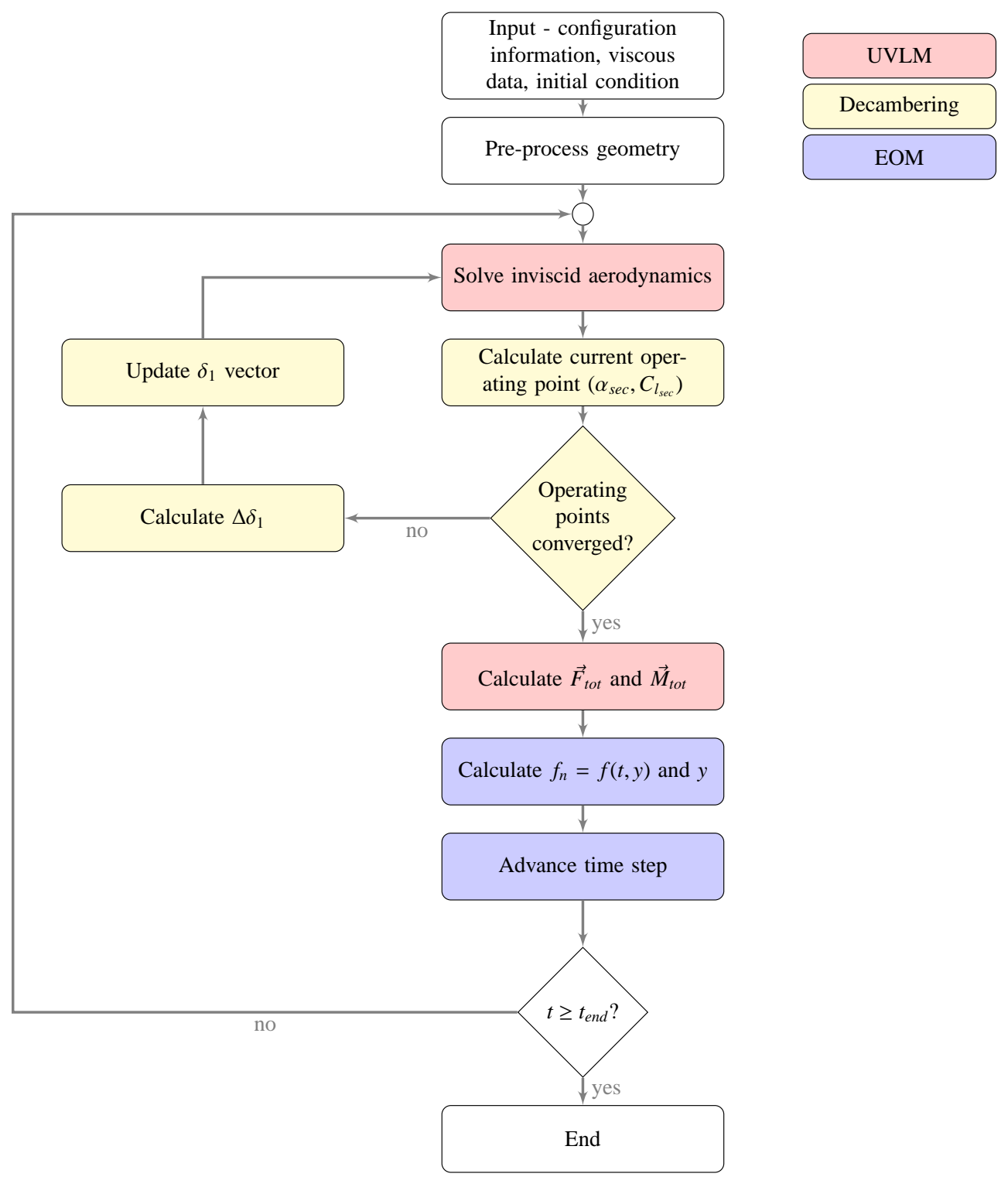

Figure 4: Flowchart for the method.

The following sections describe the steps of the combined unsteady, post-stall aerodynamics model in the order they are presented in the flowchart.

\section{A. Inputs to the aerodynamics model}

The aerodynamic model receives inputs related to the configuration, viscous data for use in the post-stall model, and initial values to facilitate time-marching the non-linear flight dynamic equations. Configurations are made up of groups of lifting surfaces. Configuration information provided includes both reference areas and geometry definitions for each individual wing. Geometry input files allow for easy definition of each wing, including support for twist, sweep, taper, and varying camber. Control surfaces geometry is also specified at this point.

Viscous $C_{l}-\alpha, C_{d}-\alpha$, and $C_{m}-\alpha$ curves are input for later use in the post-stall iteration. If control surface(s) are defined for a wing, viscous curves with controls deflected to the minimum and maximum values must also be provided. At a given time step, depending on the specified control deflection, the model will interpolate the viscous input data for wing sections that have a control. These interpolated curves will be used for the post-stall model when necessary.

Initial values of the flight dynamic state vector, $y$ are provided as input. For a full six degree of freedom, nonlinear simulation either 12 or 13 initial values are provided. The 12 element initial state vector, 
$y_{0}=\left[\begin{array}{llllllllllll}x_{e 0} & y_{e 0} & z_{e 0} & u_{0} & v_{0} & w_{0} & \phi_{0} & \theta_{0} & \psi_{0} & p_{0} & q_{0} & r_{0}\end{array}\right]^{T}$, contains initial values for inertial position, body axis velocity, body axis orientation relative to the inertial frame, and body axis angular rates. The model also allows for specification of a 13-state state vector whereby quaternions are used for attitude representation. When quaternions are used for attitude representation, initial values of the Euler angles $\left[\begin{array}{lll}\phi_{0} & \theta_{0} & \psi_{0}\end{array}\right]$ are replaced with quaternion parameters, $\left[\begin{array}{llll}\eta_{1_{0}} & \eta_{2_{0}} & \eta_{3_{0}} & \eta_{4_{0}}\end{array}\right]$

\section{B. Pre-process geometry}

Geometry data is discretized and vortex ring elements are placed on each wing as described in Section II. UVLM matrices $A_{c b}, A_{c w}, H_{l b}$, and $H_{l w}$ are computed and stored. Wake propagation matrices $C_{\Gamma b}$ and $C_{\Gamma w}$ are generated and stored at this step. As discussed in Section II, these matrices are required to enforce the Kutta-condition and for efficiently time-marching the wake vorticity. With the configuration completely defined and discretized, steady vortex lattice runs are made as described in Section III in order to calculate $\left(\frac{d C_{l}}{d \alpha}\right)_{t}$ and $\frac{d C_{l}}{d \delta_{1}}$.

\section{Solve inviscid aerodynamics}

Given the current value of the flight dynamic state vector, $y$, the pre-computed aerodynamics matrices, and the current value of decambering vector $\boldsymbol{\delta}_{1}$, the non-penetration boundary condition (Eqn. 1) and wake circulation prorogation (Eqn. 2) equations may be solved to find the gamma distribution at time step $n+1$. Equations 1 and 2 are written together, and modified to incorporate the effect of decambering, as

$$
\left[\begin{array}{l}
\boldsymbol{\Gamma}_{b} \\
\boldsymbol{\Gamma}_{w}
\end{array}\right]^{n+1}=\left[\begin{array}{cc}
A_{c b} & A_{c w} \\
0 & I
\end{array}\right]^{-1}\left\{\left[\begin{array}{cc}
0 & 0 \\
C_{\Gamma b} & C_{\Gamma w}
\end{array}\right]\left[\begin{array}{l}
\boldsymbol{\Gamma}_{b} \\
\boldsymbol{\Gamma}_{w}
\end{array}\right]^{n}+\left[\begin{array}{c}
\boldsymbol{w}+\boldsymbol{w}_{d e c} \\
0
\end{array}\right]^{n+1}\right\}
$$

Solving Eqn. 10 for the $n+1$ gamma distribution simultaneously satisfies the boundary condition and unsteady wake propagation equations while accounting for the effects of decambering on the configuration. Given the current circulation distribution acting on the configuration, the total inviscid aerodynamic loads may be computed. In the current implementation, the loads are calculated using the Kutta-Joukowski theorem, as shown in Equation 3.

\section{Calculate current operating points}

Initialization of the decambering algorithm requires knowledge of the current operating point of each 'strip' along each wing. The operating points exists in a $C_{l^{-}} \alpha$ space, and are not directly computed when inviscid aerodynamic loads are found using Kutta-Joukowski or other load calculation techniques. An important consideration when computing the operating points is that they will be compared to the appropriate input viscous $C_{l}-\alpha$ curve, which comes from 2 -D airfoil aerodynamics. Each strip is approximated as a 2-D airfoil section.

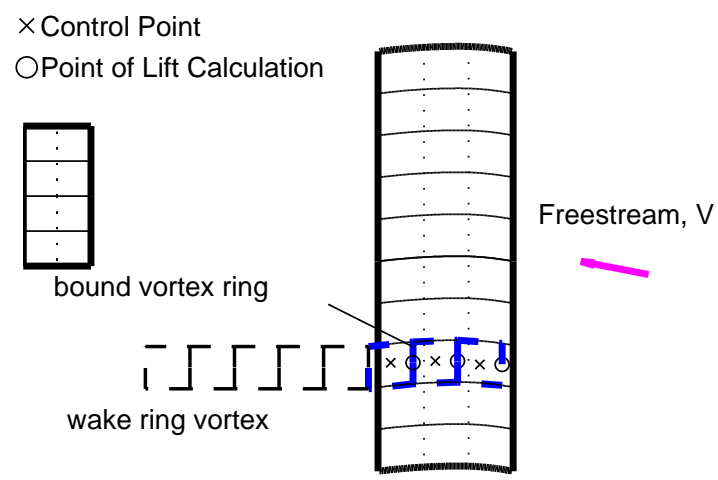

Figure 5: Simple wing-tail geometry with ring vortices (bound and wake elements) on one spanwise wing section.

In the current model, the $C_{l}$ calculation is undertaken by first calculating the total aerodynamic force acting on a 'strip'. A strip of a wing is illustrated in Figure 5 as the panel elements which have a depiction of vortex ring elements (in blue). Equation 11 shows the expression used to calculate the local $C_{l}$ values. 


$$
C_{l_{\text {sec }}}(i)=\frac{\left|\vec{F}_{c l}\right|}{1 / 2 \rho\left|\vec{V}_{\text {local }}\right|^{2} d S_{\text {strip }}}
$$

The operating $\alpha$ for each strip is calculated as given in Equation 8 .

\section{E. Check convergence of operating points}

After the operating points have been computed, they are projected onto the viscous input data along the trajectory line, as discussed in Section III. The difference between the $C_{l_{s e c}}$ and computed target $C_{l_{t}}$ is defined as the residual. If the residual is less than a pre-defined tolerance, no iteration is necessary as the points are converged. If the residual is greater than the predefined tolerance, each element of the $\boldsymbol{\delta}_{1}$ vector is altered according to Equation 12 and the aerodynamics are re-evaluated until the operating points are converged on the viscous input data.

$$
\delta_{1}^{\text {new }}(i)=\delta_{1}^{\text {old }}(i)-\frac{C_{l s}-C_{l t}}{\frac{\partial C_{l}}{\partial \delta_{1}}}
$$

\section{F. Calculate total force and moment vectors}

Inviscid aerodynamic loads are known from the steps described in Section C. After convergence of the decambering algorithm, each sectional operating point $\left(\alpha_{s e c}, C_{l_{s e c}}\right)$ falls on the viscous airfoil $C_{l}-\alpha$ curve for that section. The sectional profile drag and moment coefficients are found by querying the input $C_{d^{-}} \alpha$ and $C_{m}-\alpha$ curves at the converged operating point. For strip $i$, the contributions from these coefficients are given by Equations 13 and 14. The total contributions from all sections are added to the inviscid forces and moments according to Equations 15 and 16.

$$
\begin{gathered}
\Delta \vec{F}(i)=\frac{1}{2} \rho\left|\vec{V}_{\text {strip }}(i)\right| c(i) d S(i) C_{d_{\text {sec }}}(i)\left[\begin{array}{l}
\vec{V}_{\text {strip }}(i) \cdot \hat{i} \\
\vec{V}_{\text {strip }}(i) \cdot \hat{j} \\
\vec{V}_{\text {strip }}(i) \cdot \hat{k}
\end{array}\right] \\
\Delta \vec{M}(i)=\frac{1}{2} \rho\left|\vec{V}_{\text {strip }}(i)\right|^{2} c(i)^{2} d S(i) C_{m_{\text {sec }}}(i)\left[\begin{array}{c}
0 \\
\vec{N}_{\text {strip }}(i) \cdot-\hat{k} \\
\vec{N}_{\text {strip }}(i) \cdot \hat{j}
\end{array}\right] \\
\vec{F}_{\text {tot }}=\vec{F}_{\text {inv }}+\sum_{i} \Delta \vec{F}(i) \\
\vec{M}_{t o t}=\vec{M}_{i n v}+\sum_{i}(\vec{r} \times \Delta \vec{F}(i))
\end{gathered}
$$

where

$$
\vec{r}=\left[\begin{array}{lll}
x_{q c}-x_{C G} & y_{q c}-y_{C G} & z_{q c}-z_{C G}
\end{array}\right]
$$




\section{G. Time marching of the equations of motion}

For a full nonlinear 6 degree of freedom simulation (using quaternion parameters), Equation 17 provides the flight dynamic state vector derivative, $f_{n}$.

$$
\begin{aligned}
\dot{x} & =u\left(\eta_{4} \eta_{4}+\eta_{1} \eta_{1}-\eta_{2} \eta_{2}-\eta_{3} \eta_{3}\right)+v\left(2 \eta_{2} \eta_{1}-2 \eta_{4} \eta_{3}\right)+w\left(2 \eta_{3} \eta_{1}+2 \eta_{4} \eta_{2}\right) \\
\dot{y} & =u\left(2 \eta_{1} \eta_{2}+2 \eta_{4} \eta_{3}\right)+v\left(\eta_{4} \eta_{4}-\eta_{1} \eta_{1}+\eta_{2} \eta_{2}-\eta_{3} \eta_{3}\right)+w\left(2 \eta_{3} \eta_{2}-2 \eta_{4} \eta_{1}\right) \\
\dot{z} & =u\left(2 \eta_{1} \eta_{3}-2 \eta_{4} \eta_{2}\right)+v\left(2 \eta_{2} \eta_{3}+2 \eta_{4} \eta_{1}\right)+w\left(\eta_{4} \eta_{4}-\eta_{1} \eta_{1}-\eta_{2} \eta_{2}+\eta_{3} \eta_{3}\right) \\
\dot{u} & =r v-q w+g\left(2 \eta_{1} \eta_{3}-2 \eta_{4} \eta_{2}\right)+\left(X_{\text {tot }}+\text { Xthrust }\right) / \text { mass } \\
\dot{v} & =-r u+p w+g\left(2 \eta_{2} \eta_{3}+2 \eta_{4} \eta_{1}\right)+Y_{\text {tot }} / \text { mass } \\
\dot{w} & =q u-p v+g\left(\eta_{4} \eta_{4}-\eta_{1} \eta_{1}-\eta_{2} \eta_{2}+\eta_{3} \eta_{3}\right)+Z_{\text {tot }} / \text { mass } \\
\dot{\eta_{1}} & =1 / 2\left(\eta_{4} p-\eta_{3} q+\eta_{2} r\right) \\
\dot{\eta}_{2} & =1 / 2\left(\eta_{3} p+\eta_{4} q-\eta_{1} r\right) \\
\dot{\eta_{3}} & =1 / 2\left(-\eta_{2} p+\eta_{1} q+\eta_{4} r\right) \\
\dot{\eta}_{4} & =1 / 2\left(-\eta_{1} p-\eta_{2} q-\eta_{3} r\right) \\
\dot{p} & =\left(c_{1} r+c 2 p-c_{4} h_{\text {eng }}\right) q+\left(c_{3} \text { Roll }_{\text {tot }}+c_{4} N_{\text {tot }}\right) \\
\dot{q} & =\left(c_{5} p+c_{7} h_{\text {eng }}\right) r-c_{6}\left(p^{2}-r^{2}\right)+c_{7} M_{t o t} \\
\dot{r} & =\left(c_{8} p-c_{2} r-c_{9} h_{\text {eng }}\right) q+\left(c_{4} \text { Roll }_{\text {tot }}+c_{9} N_{\text {tot }}\right)
\end{aligned}
$$

where the following constants have been defined [25]:

$$
\begin{aligned}
c_{1} & =\frac{\left(I_{y}-I_{z}\right) I_{z}-I_{x z}^{2}}{I_{x} I_{z}-I_{x z}^{2}} & c_{2} & =\frac{\left(I_{x}-I_{y}+I_{z}\right) I_{x z}}{I_{x} I_{z}-I_{x z}^{2}} \\
c_{3} & =\frac{I_{z}}{I_{x} I_{z}-I_{x z}^{2}} & c_{4} & =\frac{I_{x z}}{I_{x} I_{z}-I_{x z}^{2}} \\
c_{5} & =\frac{I_{z}-I_{x}}{I_{y}} & c_{6} & =\frac{I_{x z}}{I_{y}} \\
c_{7} & =\frac{1}{I_{y}} & c_{8} & =\frac{\left(I_{x}-I_{y}\right) I_{x}-I_{x z}^{2}}{I_{x} I_{z}-I_{x z}^{2}} \\
c_{9} & =\frac{I_{x}}{I_{x} I_{z}-I_{x z}^{2}} & h_{\text {eng }} & =\left[\begin{array}{lll}
I_{p} \Omega_{p} & 0 & 0
\end{array}\right]
\end{aligned}
$$

The flight dynamic state vector, $y$, is updated using a numerical method for the solution of differential equations. In the current methodology, only explicit methods are considered since only previous values of the state time history are available. Flight dynamic simulation results presented in this paper have all been computed using the fourth-order Adams-Bashforth method, whose state update equation is given by Equation 18 [26].

$$
y_{n+1}=y_{n}+\frac{d t}{24}\left(55 f_{n}-59 f_{n-1}+37 f_{n-2}-9 f_{n-3}\right)
$$

The fourth-order Adams-Bashforth method requires that a starter formula is used to generate the first few points. In this work forward Euler is used to generate these points.

\section{Results}

The methods presented above for flight dynamics simulation with unsteady and post-stall aerodynamics are exercised next for a high altitude long endurance unmanned aerial vehicle (HALE UAV) and a light general aviation aircraft

\section{A. Unsteady aerodynamic effects on aircraft flight dynamics}

This section demonstrates the impact of unsteady aerodynamic effects on aircraft flight dynamics. In particular, it illustrates how the short-period mode cannot be accurately predicted through a quasi-steady approximation. To that goal, the linearized unsteady vortex lattice described in Section II will be compared to a quasi-steady formulation of the method, as well as to classical (quasi-steady) approximations for longitudinal rigid-body modes. Hence, fully attached flow is assumed, that is, post-stall modeling is not included in this set of results. An aircraft configuration 


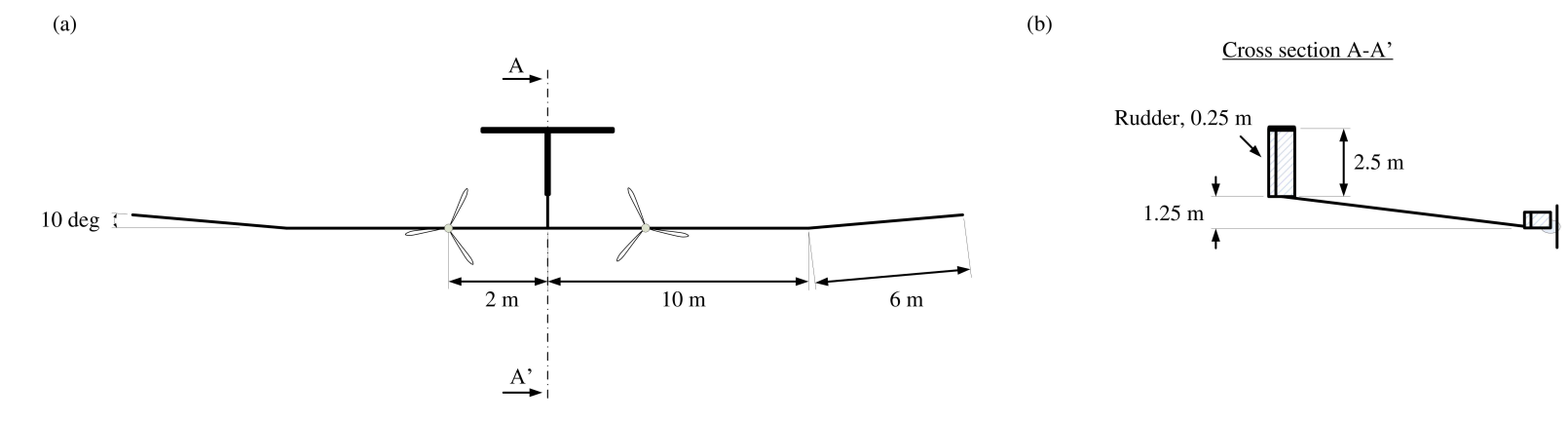

(b)

(c)

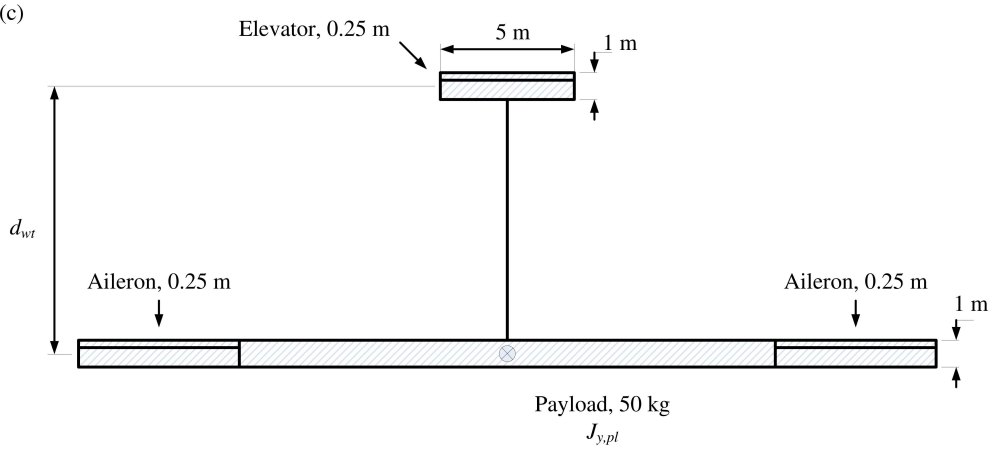

(d)

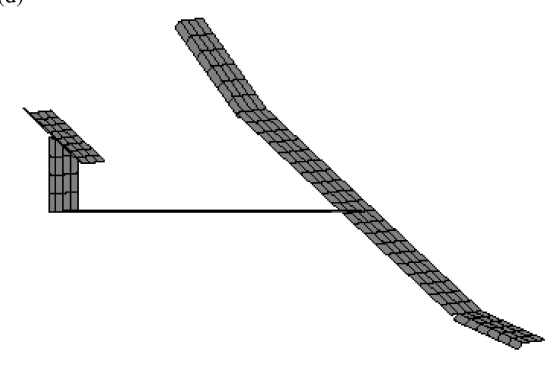

Figure 6: Test aircraft geometry (not to scale): (a) front view, (b) cross section, (c) top view, and (d) 3-D view of the aircraft showing deployed control surfaces.

representative of a HALE UAV will be used, shown in Figure 6, which was loosely based on that proposed in Ref. [27] but has been modified to resemble the latest version of QinetiQ's Zephyr.

The vehicle consists of a very large aspect ratio wing, fuselage and empennage, and the distance between the quarter chords of wing and tail, $d_{w t}$ is taken as parameter. All members are assumed to be rigid (no structural deformations). The main wing encompasses rigidly-linked members, with a 10 deg dihedral angle at both ends. The outboard winglets include full-span trailing-edge ailerons of a quarter-chord length. The horizontal stabilizer and the vertical fin are modeled as lifting surfaces, and they also include quarter-chord-length control surfaces, elevator and rudder, respectively. The fuselage, in turn, is represented by a non-lifting beam, and it is inclined so that the empennage is raised with respect to the main wing. This feature is important in order to guarantee that the wake shed by the main wing does not impinge on the tail - the UVLM can provide some insight into wake-tail collisions [19], but this is a scenario to be avoided. All lifting surfaces are uncambered, untwisted, unswept, and untapered. The aircraft carries a payload of $50 \mathrm{~kg}$ at the root of the main wing (the only non-structural mass of the problem, modeled as a point load with pitch inertia $J_{y, p l}$ ), and is powered by two massless propellers, which are modeled as point forces rigidly linked to the wing.

The relevant properties of the members are listed in Table 1. The mass per unit length of the fuselage is the same as that of the horizontal tailplane (HTP) and vertical tailplane (VTP), and thus the total mass of this aircraft, including payload and structural mass, is $M=75.4 \mathrm{~kg}$. It is assumed that the aircraft flies at $V_{\infty}=30 \mathrm{~m} / \mathrm{s}$ at an altitude of $20 \mathrm{~km}$, where the density is $\rho_{\infty}=0.0899 \mathrm{~kg} / \mathrm{m}^{3}$. Throughout the subsequent numerical studies using vortex lattice aerodynamics, a nondimensional time step of $\Delta t^{*}=V_{\infty} \Delta t / c=0.25$ has been used, where $c$ is the characteristic chord length. All lifting surfaces have been discretized using 4 panels chordwise. 32 spanwise panels are used in the main wing, 8 in the HTP, and 4 in the VTP. The wake is truncated after 20 chord lengths.

First of all, the aircraft is trimmed for steady level flight (in undisturbed conditions) through three inputs, namely angle of attack, AoA, elevator deflection, $\delta_{e}$, and thrust per propeller, $T$ - the rest of control surfaces, ailerons and rudder, remain undeployed. The trim iterations follow the standard Newton-Raphson method, using finite differences to compute the Jacobian. The inputs required to trim the aircraft as a function of the distance between wing and tail, $d_{w t}$, are shown in Figure 7. It can be observed that $d_{w t}$ has a minor effect on the necessary thrust, but if affects the angle of attack, and more significantly, the elevator deflection. The center of mass of the configuration falls very close to the payload location, and a substantial contribution to the pitching moment is due to the horizontal HTP. In order to counteract the anticlockwise moment created by the surface, the elevator needs a negative deflection to introduce 
Table 1: Test aircraft properties.

\begin{tabular}{llll}
\hline \hline & Main wing & HTP & VTP \\
\hline Chord & $1 \mathrm{~m}$ & $1 \mathrm{~m}$ & $1 \mathrm{~m}$ \\
Span & $32 \mathrm{~m}$ & $5 \mathrm{~m}$ & $2.5 \mathrm{~m}$ \\
Center of gravity & $50 \%$ chord & $50 \%$ chord & $50 \%$ chord \\
Mass per unit length & $0.75 \mathrm{~kg} / \mathrm{m}$ & $0.08 \mathrm{~kg} / \mathrm{m}$ & $0.08 \mathrm{~kg} / \mathrm{m}$ \\
Moment of inertia & $0.1 \mathrm{~kg} \cdot \mathrm{m}$ & $0.01 \mathrm{~kg} \cdot \mathrm{m}$ & $0.01 \mathrm{~kg} \cdot \mathrm{m}$ \\
\hline \hline
\end{tabular}

clockwise pitching. The farther apart the wing and the tail are, the larger the anticlockwise moment due to the HTP (longer arm) and thus the larger the required elevator deployment (in absolute value).
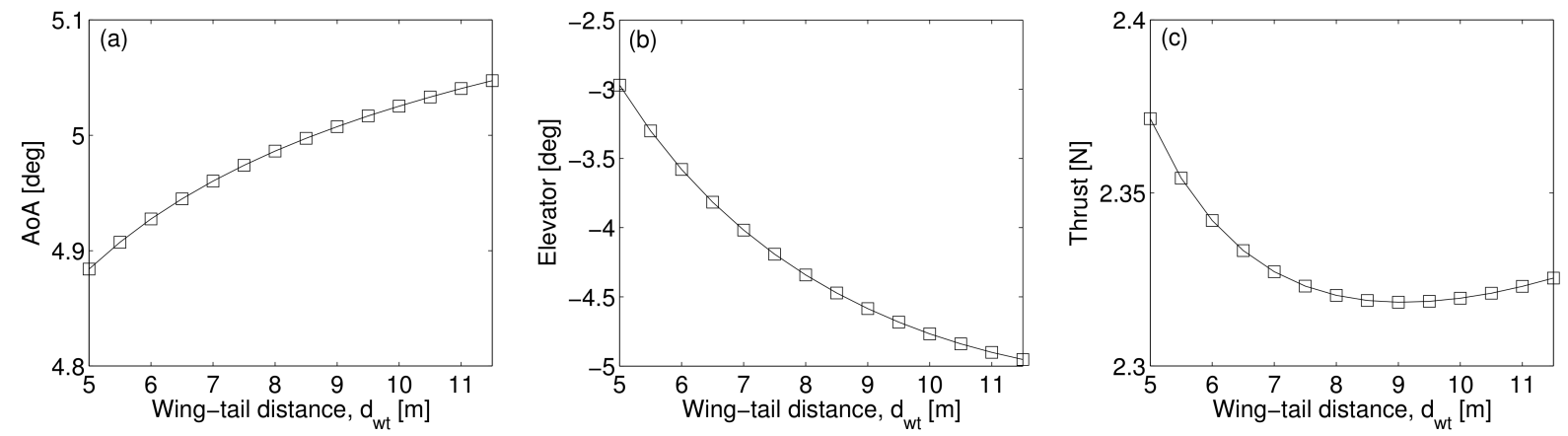

Figure 7: Trim inputs for rigid HALE UAV with $J_{y, p l}=0$ at $V_{\infty}=30 \mathrm{~m} / \mathrm{s}$ as a function of wing-tail distance: (a) angle of attack, (b) elevator deflection, and (c) thrust.

Once the trim conditions have been determined, a linear stability analysis is carried out to determine the flight dynamics modes of the vehicle. This is accomplished using the framework SHARP [5], by linearizing the equations of motion of the rigid aircraft about the corresponding trim conditions through small perturbation analysis, with the unsteady aerodynamic loads obtained from the vortex lattice. This leads to a monolithically coupled linear state space system of the form $\dot{\mathbf{x}}=A \mathbf{x}$, where the state vector $\mathbf{x}$ includes circulation states and derivatives from the vortex lattice, as well as flight velocities and orientation. The stability of the system can be determined directly from an eigenvalue analysis of the matrix $A$. More details on the small perturbation analysis with vortex lattice aerodynamics and the algebra leading to the state space equations can be found in Ref. [5], whereby a geometrically exact beam model and Euler angles are used to describe the aircraft dynamics, and the resulting system is formulated in discrete time. For a rigid aircraft, the approach is equivalent to performing the small perturbation analysis on Eqs. (17);

The flight dynamics modes obtained with the unsteady vortex lattice, denoted as $U V L M$, will be compared to different quasi-steady approximations. First of all, a quasi-steady vortex lattice can be considered. In this case, the changes in circulation strength on the bound trailing edge panels are propagated instantaneously along the whole wake length, that is, $C_{\Gamma w}^{q s}=0$ in Eq. (2), and $C_{\Gamma b}^{q s}$ contains ones in all relevant elements instead of the zeros of the unsteady counterpart. This quasi-steady approach, designated as $Q V L M$, is equivalent to using horseshoe vortices at each spanwise station (strip) of the lifting surfaces.

Both UVLM and QVLM account for lateral-directional as well as longitudinal modes. An additional quasi-steady approximation can be derived for longitudinal modes based on classical flight dynamics. Consider a trimmed aircraft following a rectilinear flight path with constant forward velocity $V_{x}$ and inclined at an angle $\Theta$ above the horizontal (its reference climb angle). From the small perturbation equations of motion about that equilibrium point [28, p. 175] and assuming quasi-steady aerodynamic loads, one has for the quasi-steady longitudinal problem

$$
\dot{\mathbf{x}}_{q s, l}=A_{q s, l} \mathbf{x}_{q s, l},
$$


with $\dot{\mathbf{x}}_{q s, l}=A_{q s, l} \dot{\mathbf{x}}$, with $x_{q s, l}^{T}=\left\{\begin{array}{llll}v_{x} & v_{z} & \omega_{y} & \theta\end{array}\right\}$, and

$$
A_{q s, l}=\left[\begin{array}{cccc}
2 \frac{M g \sin \Theta-T}{M V_{x}} & \frac{g \cos \Theta}{V_{x}}\left(1-\frac{2 S C_{L, \alpha}}{\pi b^{2} e}\right) & 0 & -g \cos \Theta \\
-\frac{2 g \cos \Theta}{V_{x}} & \frac{M g \sin \Theta-T}{M V_{x}}-\frac{\rho_{\infty} V_{x} S C_{L, \alpha}}{2 M} & V_{x}-\frac{\rho_{\infty} V_{x} S_{t} l_{t} C_{L, \alpha}^{t}}{2 M} & -g \sin \Theta \\
0 & \frac{\rho_{\infty} V_{x}\left(S l_{w} C_{L, \alpha}^{w}-S_{t} l_{t} C_{L, \alpha}^{t}\right)}{2 J_{y}} & -\frac{\rho_{\infty} V_{x} S_{t} l_{t}^{2} C_{L, \alpha}^{t}}{2 J_{y}} & 0 \\
0 & 0 & 1 & 0
\end{array}\right],
$$

whereby stability axes have been employed. $J_{y}$ is the pitch inertia of the full aircraft, $S, c, b$ represent planform area, mean aerodynamic chord, and wing span respectively, $e=0.8$ is the efficiency factor for a rectangular wing, $g=9.81$ $\mathrm{m} / \mathrm{s}^{2}$ is the gravitational acceleration, $l$ are distances between aircraft center of mass and lifting-surface quarter chord, and sub- and super-scripts $w$ and $t$ refer to wing and tail, respectively. More details of the derivation of Eq. (20) can be found in Ref. [23, §Appendix D].

The eigenvalue problem derived from Eq. (20) allows the dominant longitudinal modes of the rigid aircraft to be obtained. If the eigenvalue problem is solved analytically, the characteristic polynomial corresponds to the longitudinal stability quartic. For comparison with the unsteady and quasi-steady methodologies based on the vortex lattice, the same thrust input is used, the climb angle is set to $\Theta=0$, and the relevant aerodynamic coefficients, $C_{L, \alpha}$, are estimated from a steady vortex lattice. Note that this approximation assumes that the tail lift acts in the same direction as the main wing, which neglects the contributions of the downwash to the instantaneous wind velocity vector of the tail; in other words, the downwash induced by the wake on the tail lift coefficient is included only in a quasi-steady manner. In addition, the contribution of the pitch rate, $\omega_{y}$, to the instantaneous wind velocity vector of the tail is also neglected, a further quasi-steady approximation. This approach will be denoted Classical. It should be noted that the effects neglected by the quasi-steady approximations are often incorporated in flight dynamics by introducing lag terms, which have not been included in this implementation.

Figure 8 presents the dominant modes of the aircraft comparing unsteady aerodynamics and quasi-steady approximations. The damping of all modes is negative, but the absolute value is plotted. UVLM and QVLM provide as many modes as states has the system (of the order of thousands), but most correspond to highly damped purely aerodynamic poles, and thus only the dominant phugoid, dutch roll and short period are presented. In turn, the quasi-steady approximation for longitudinal modes provides only four poles, which in general correspond to two pairs of complex conjugates, representing phugoid and short period modes. For this particular configuration, however, the quasi-steady approximations do not predict the short period mode and two real negative roots are obtained instead - the aircraft is pitch stable. As its frequency is higher, the short period mode is more heavily affected by the unsteady aerodynamics and both quasi-steady approximations fail to capture it. For the highest values of $d_{w t}$, the short period mode also disappears from the unsteady predictions. For the phugoid and the dutch roll, the QVLM agrees remarkably well with the UVLM, illustrating that a quasi-steady approximation does work well for these modes. The mismatch between Classical method an unsteady aerodynamics for the phugoid mode is higher, but still reasonable (below 7\% error), and only at very low wing-tail distances is there qualitative discrepancy. This indicates that the Classical quasi-steady method is indeed a sound approximation for the phugoid mode. 

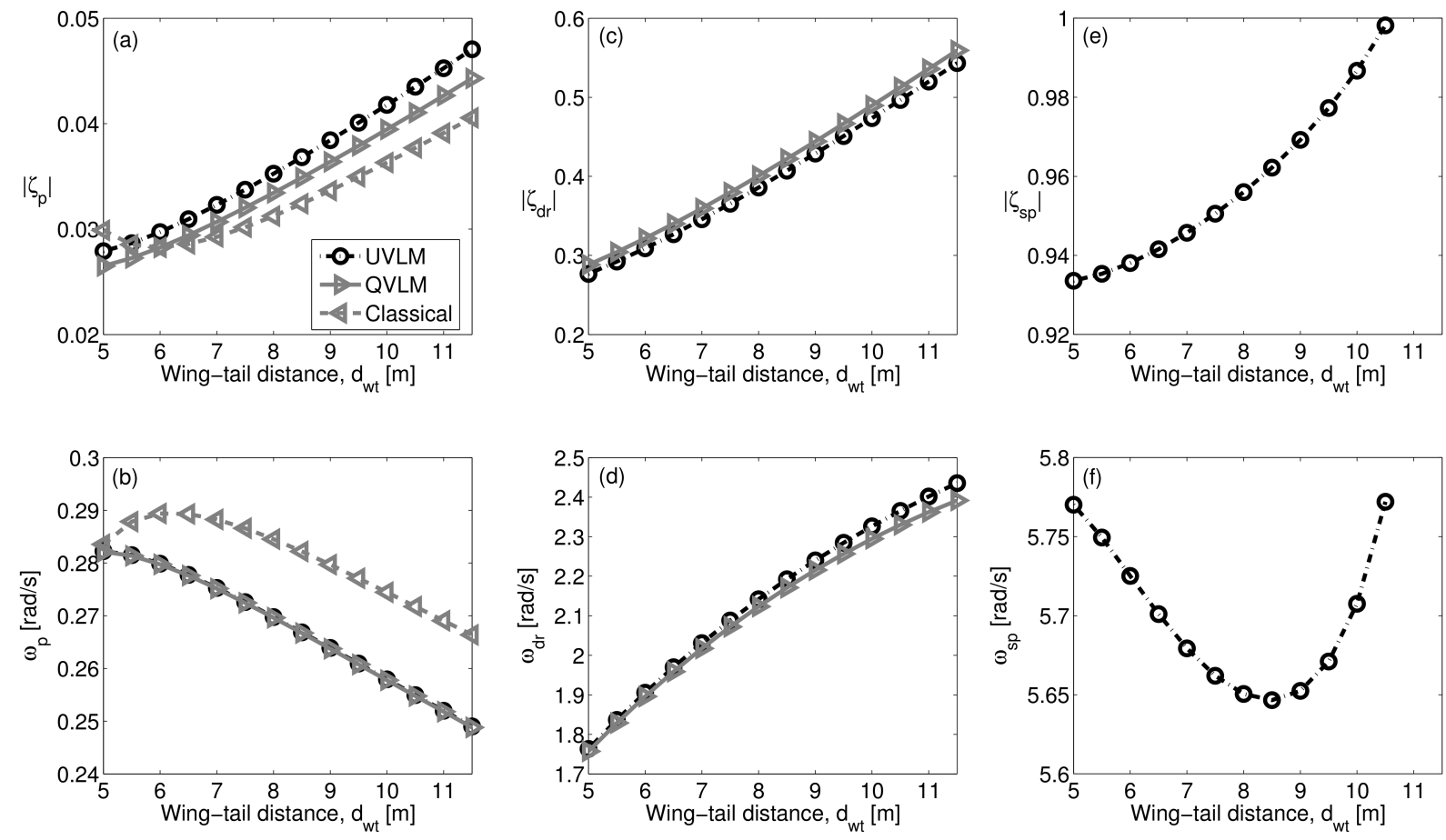

Figure 8: Comparison of dominant flight dynamics modes using unsteady aerodynamic and quasi-steady approximations $\left(J_{y, p l}=0, V_{\infty}=30 \mathrm{~m} / \mathrm{s}\right.$ ): (a) phugoid damping, (b) phugoid frequency, (c) dutch roll damping, (d) dutch roll frequency, (e) short period damping, and (f) short period frequency.

In order to try to capture a short-period mode with the quasi-steady approximations, an additional pitch inertia is included, $J_{y, p l}=150 \mathrm{~kg} \cdot \mathrm{m}^{2}$, which modifies the aircraft characteristics. The exact same procedure is followed: the aircraft is trimmed first and the flight dynamics modes are computed after linearizing the equations of motion about each trim condition. Figure 9 displays the inputs required to trim the aircraft as a function of $d_{w t}$. The curves are analogous to those of the original configuration presented in Figure 7, but the numerical values obviously change.
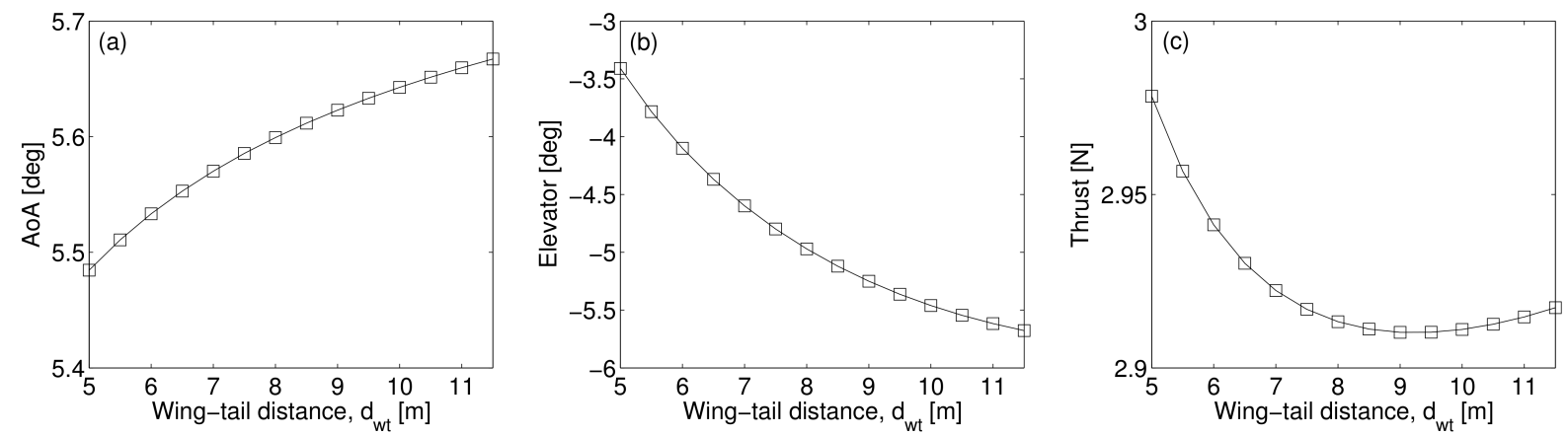

Figure 9: Trim inputs for rigid HALE UAV with additional pitch inertia $\left(J_{y, p l}=150 \mathrm{~kg} \cdot \mathrm{m}^{2}\right)$ at $V_{\infty}=30 \mathrm{~m} / \mathrm{s}$ as a function of wing-tail distance: (a) angle of attack, (b) elevator deflection, and (c) thrust.

The phugoid, dutch roll and short period of the modified configuration are presented in Figure 10. Once again, the agreement between QVLM and UVLM is excellent in the phugoid and dutch roll cases. The Classical approximation is also notably close across a wide range of $d_{w t}$, and only at very low wing-tail distances does it show some qualitative discrepancy in phugoid frequency - still, the maximum error is below 4\%. For this aircraft characteristics, the quasisteady approximations do predict a short period mode except for large values of $d_{w t}$, which is consistent with the UVLM. However, in addition to the more significant quantitative discrepancy (reaching up to $30 \%$ error), there is a clear qualitative departure between quasi-steady and unsteady curves, particularly in frequency. 

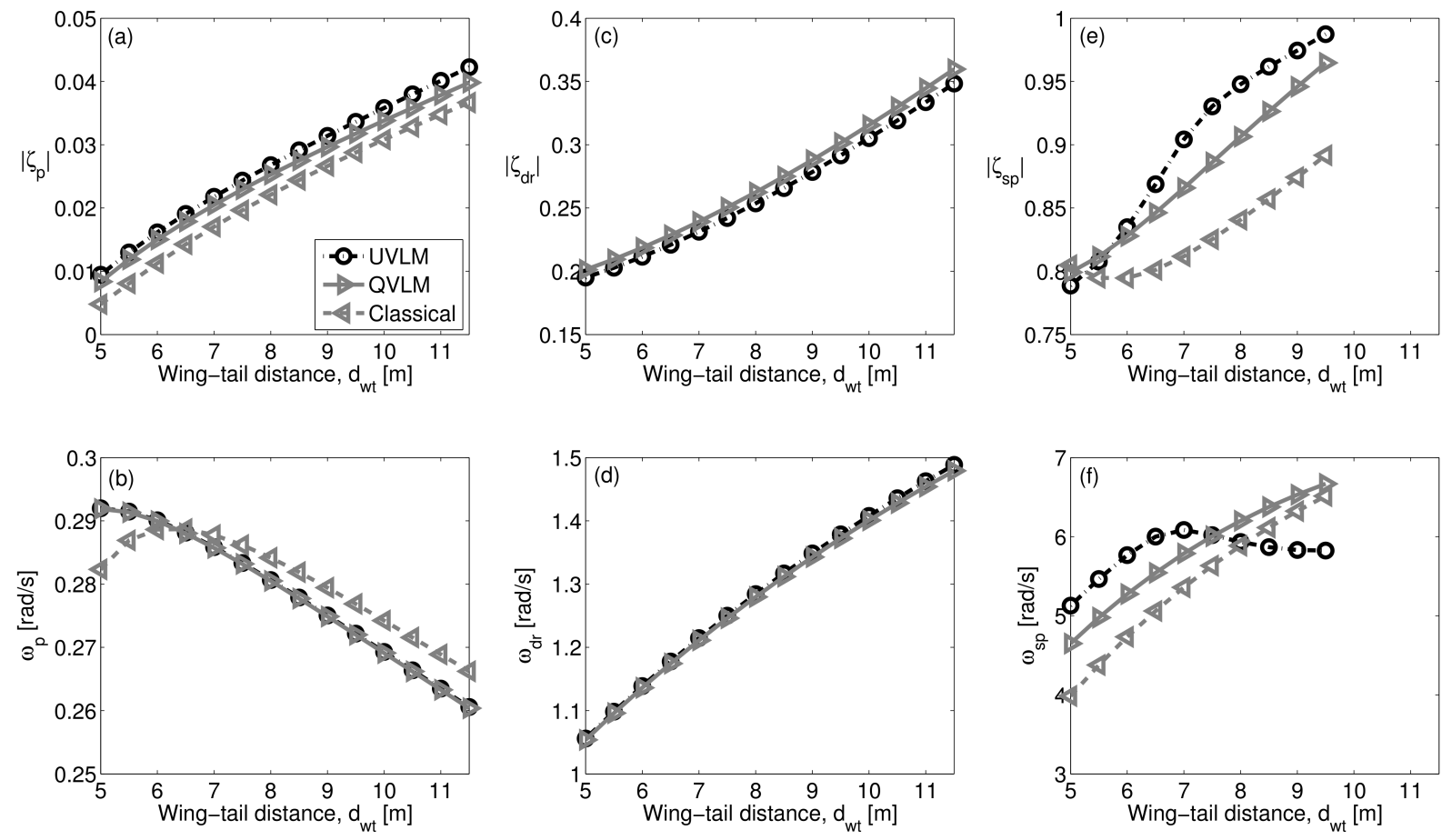

Figure 10: Comparison of dominant flight dynamics modes using unsteady aerodynamic and quasi-steady approximations with additional pitch inertia $\left(J_{y, p l}=150 \mathrm{~kg} \cdot \mathrm{m}^{2}, V_{\infty}=30 \mathrm{~m} / \mathrm{s}\right)$ : (a) phugoid damping, (b) phugoid frequency, (c) dutch roll damping, (d) dutch roll frequency, (e) short period damping, and (f) short period frequency.

The remarkable insight and even accuracy offered by classical approximations, developed when current computational resources could not be dreamt of, deserves immense credit. However, the above studies exemplify the errors incurred when using quasi-steady approximations for flight dynamics predictions, specifically on the short period mode. Furthermore, higher frequency modes cannot be captured with these methods. While this might not be essential in rigid-body flight dynamics, it is critical when realistic flexible aircraft, which exhibit higher frequency structural modes, are considered, warranting unsteady aerodynamic modeling.

\section{B. Post-Stall Modeling}

This section presents simulation results that demonstrate the capability the post-stall model offers for flight dynamics simulation. Results were generated using a rigid aircraft geometry, the unsteady aerodynamics model, and the fully non-linear time-marching scheme for the flight dynamics. The test configuration in this example is loosely adopted from a light general aviation aircraft from Roskam [29]. The main wing is rectangular with a camberline from a NACA4415 airfoil and $2^{\circ}$ dihedral. The horizontal and vertical tail planes are also rectangular lifting surfaces with a symmetric airfoil cross-sections, and feature full span control surfaces of quarter-chord-length. The wings in this example have a constant chord with no aerodynamic twist, thus the input viscous airfoil data that is assigned to each section is constant throughout each wing. The viscous input data with zero-flap deflection used in the simulation is obtained from computational fluid dynamics simulation, described in Reference 30. The sectional viscous data used when considering control deflections were generated by combining predicted coefficients from XFOIL [31] which were merged with the high $\alpha$ data beyond the convergence capability of XFOIL. Input viscous airfoil data for each airfoil used in the post-stall test case is shown in Figures 11 and 12. Relevant configuration details are presented in Table 2, and mass properties and reference areas are given in Table 3. 

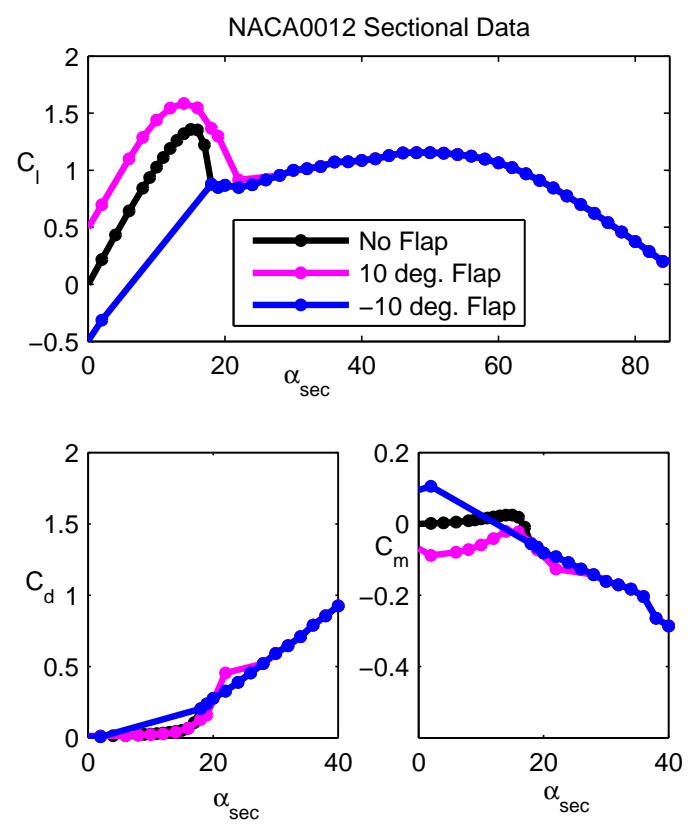

Figure 11: Input viscous data for NACA0012 airfoil.
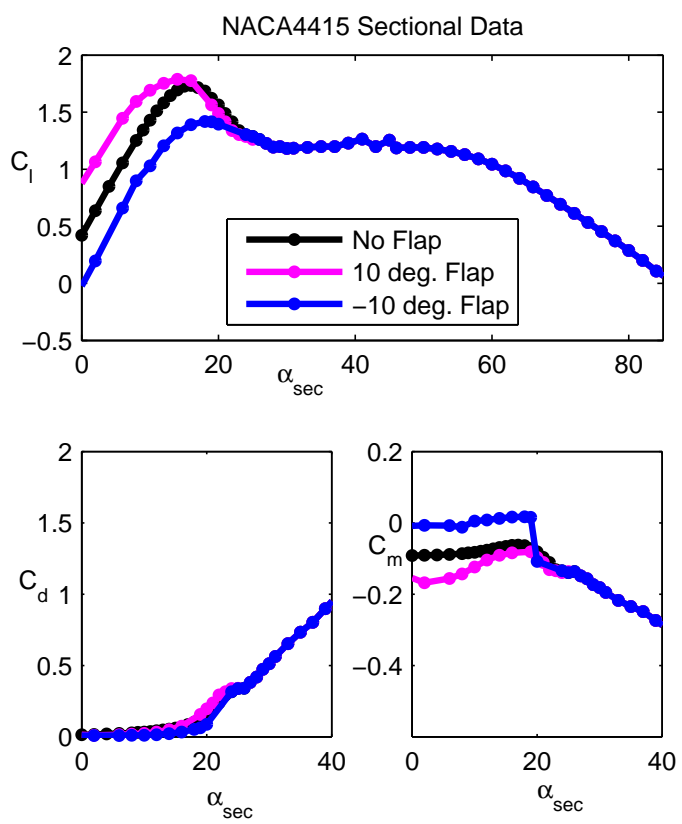

Figure 12: Input viscous data for NACA4415 airfoil.

Table 2: Post-stall example aircraft geometric properties.

\begin{tabular}{llll}
\hline \hline Geometry & Main wing & HTP & VTP \\
\hline Chord & $1.5 \mathrm{~m}$ & $1 \mathrm{~m}$ & $1 \mathrm{~m}$ \\
Span & $11 \mathrm{~m}$ & $3.5 \mathrm{~m}$ & $1.8 \mathrm{~m}$ \\
Airfoil Cross Section & NCAA4415 & Symmetric & Symmetric
\end{tabular}

Table 3: Post-stall example aircraft mass properties and reference areas.

\begin{tabular}{lll}
\hline Mass & 1200 & $\mathrm{~kg}$ \\
$I_{x}$ & 1285 & $\mathrm{~kg}-\mathrm{m}^{2}$ \\
$I_{y}$ & 1824 & $\mathrm{~kg}-\mathrm{m}^{2}$ \\
$I_{z}$ & 2666 & $\mathrm{~kg}-\mathrm{m}^{2}$ \\
$I_{X Z}$ & 0 & $\mathrm{~kg}-\mathrm{m}^{2}$ \\
$S_{r e f}$ & 16.5 & $\mathrm{~m}^{2}$ \\
$b_{r e f}$ & 11.0 & $\mathrm{~m}$ \\
$c_{r e f}$ & 1.5 & $\mathrm{~m}$ \\
$X_{c g}$ & -0.7 & $\mathrm{~m}$ \\
$X_{N P}$ & -0.9 & $\mathrm{~m}$ \\
\hline
\end{tabular}

For the simulation example, the initial speed of the aircraft is set at $V_{\infty}=40 \mathrm{~m} / \mathrm{s}$ at sea level, where the density is $\rho_{\infty}=1.225 \mathrm{~kg} / \mathrm{m}^{3}$. A time step of $\Delta t=0.02 \mathrm{~s}$, corresponding to an initial non dimensional time step of $\Delta t^{*}=$ $V_{\infty} \Delta t / c=0.53$, was used throughout the simulation and was found to be sufficiently small. The configuration was discretized using two panels chordwise, with 20 spanwise panels in the wing and 8 in the tail surfaces.

As seen in the trajectory drawn as Figure 13, the simulation example begins with the aircraft gliding near a trim condition at $V_{\infty}=40 \mathrm{~m} / \mathrm{s}$ traveling in the positive $x$ direction. After $0.4 \mathrm{~s}$ of simulation time, the elevator is deflected from the trim condition to pitch the aircraft up. Figure 14 shows an immediate response to the up elevator command in the $\alpha$ signal. After this immediate reaction to the elevator deflection, the angle of attack gradually increases as the elevator input is held until the aircraft stalls. A post-stall lift distribution from simulation time $=7.6 \mathrm{~s}$ is given in Figure 16. The post-stall lift distribution shown is asymmetric, as around the same time the stall is reached the rudder 
is deflected to its maximum value, as can be seen by the plot of the rudder command and $\beta$ signal in Figure 15 . The stall is not maintained for long - the aircraft pitches down nearly immediately after the stall. Control inputs are relaxed and the aircraft proceeds to enter a spiral like motion. A lift distribution from a point in the spiral motion is shown in Figure 17.

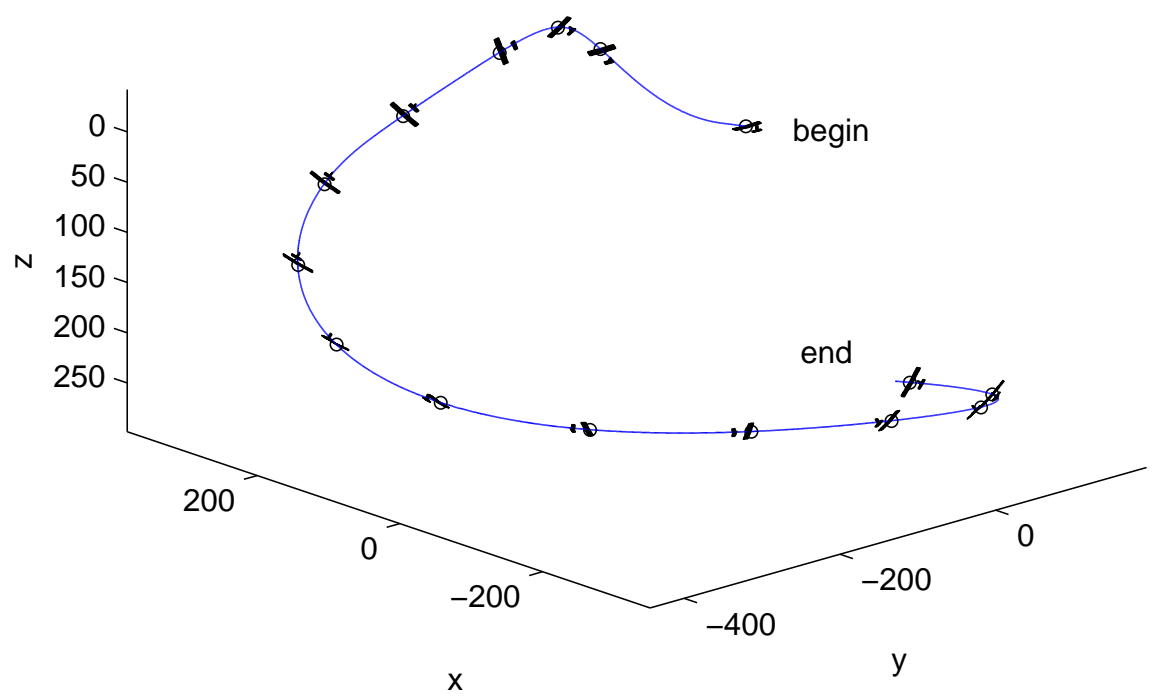

Figure 13: Post-stall simulation example trajectory.
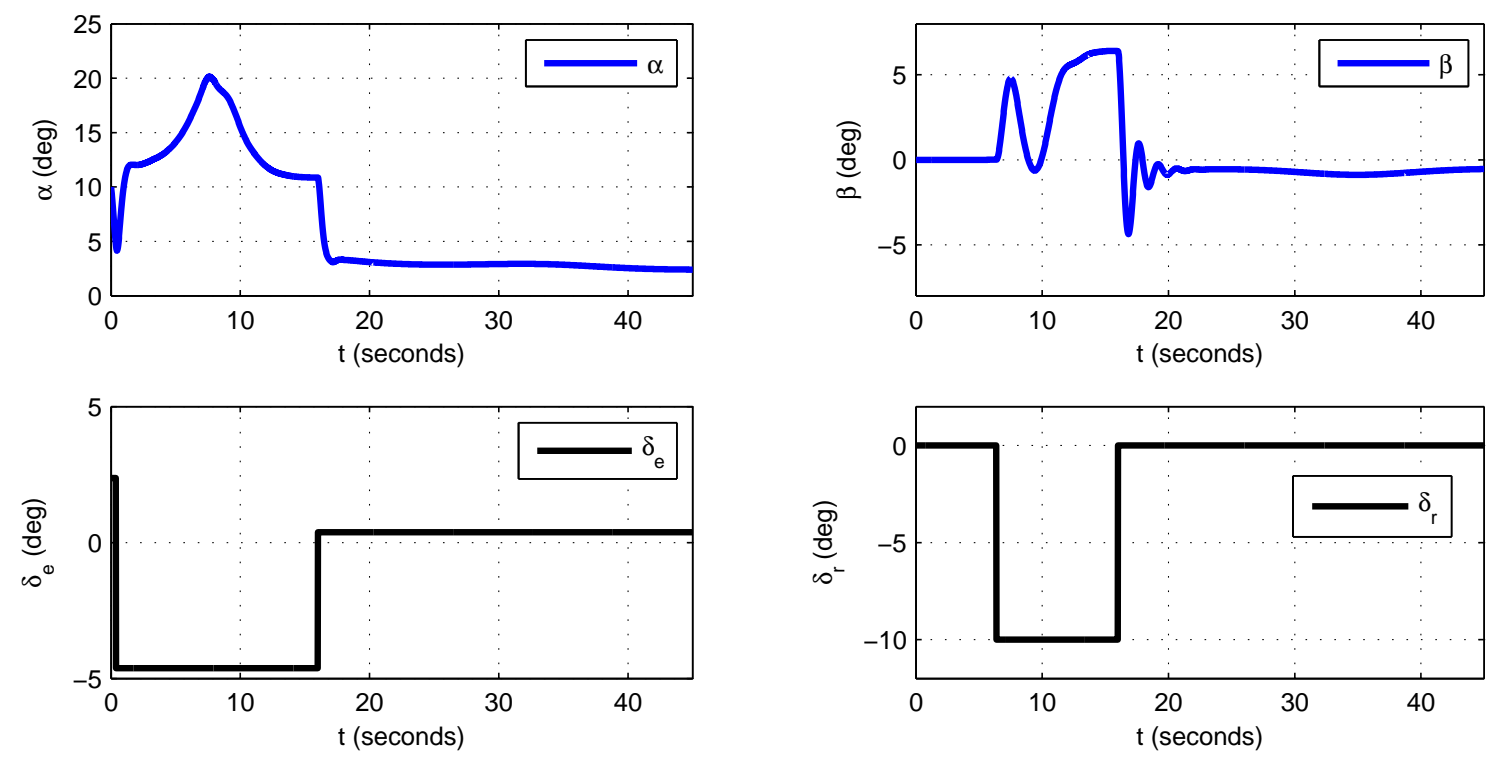

Figure 14: Elevator command and $\alpha$ response from the post-stall simulation example.

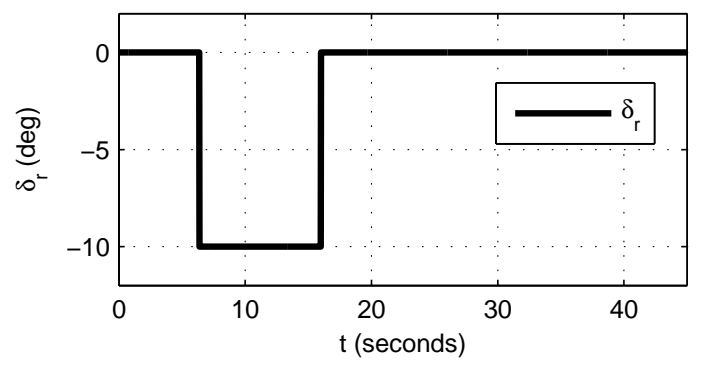

Figure 15: Rudder command and $\beta$ response from the post-stall simulation example. 


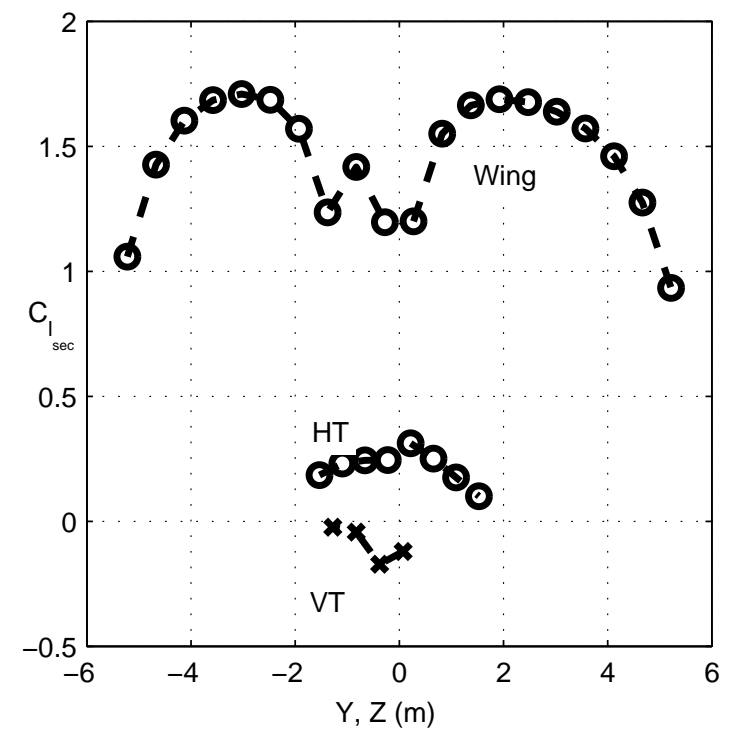

Figure 16: Post-stall $C_{l}$ distribution at Sim Time $=$ 7.6s.

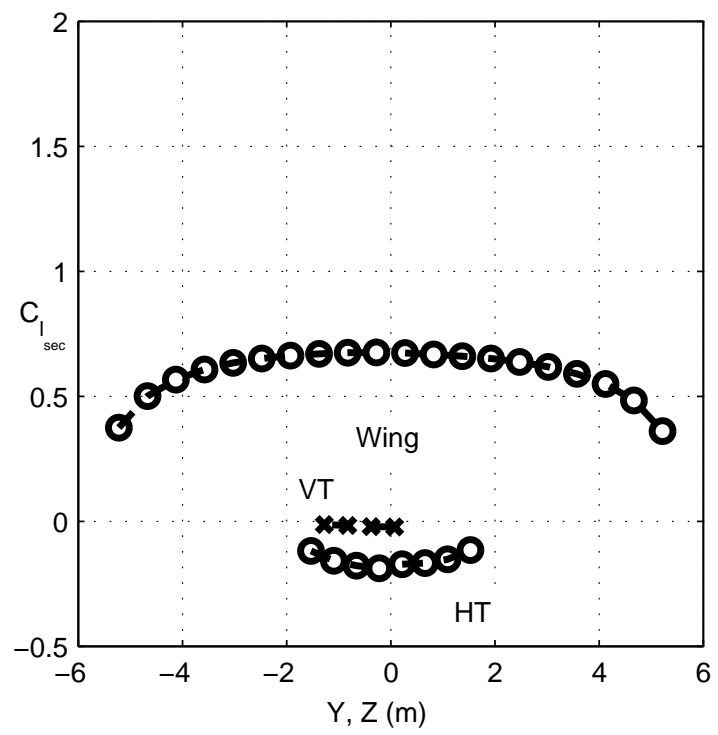

Figure 17: Unstalled $C_{l}$ distribution from Sim Time $=$ 20 s.

\section{Conclusions}

Flight at high angles of attack can be a design decision due to mission requirements, or it may be an undesirable outcome of flight maneuvers, structural deformations and/or atmospheric disturbances. In all cases, the likelihood of operating beyond the stall boundary requires models that account for the nonlinear aerodynamic characteristics. In this context, approximate albeit fast methods that permit aerodynamics-in-the-loop might offer superior capabilities to look-up tables for aircraft stability, control and simulation, especially at conceptual design stages.

This paper has presented a low-order method oriented towards that goal, combining 3D unsteady vortex lattice aerodynamics with a decambering correction to model post-stall behavior and integrating them with the aircraft equations of motion. The unsteady vortex lattice method, based on potential flow theory, is linearized in a body-fixed frame assuming a frozen wake geometry, a valid approximation as long as the aircraft maneuvers are not too fast. The linearization only requires the evaluation of aerodynamic influence coefficients at a preprocessing step, leading to a very efficient numerical method for the prediction of unsteady, inviscid aerodynamics. The decambering approach incorporates post-stall modeling, when necessary, through the rotation of normal vectors to match $2 \mathrm{D}$ viscous data, readily available from XFOIL or similar. This correction is naturally appended into the vortex lattice and does not require the recomputation of influence coefficients. The decambering is applied in strip-theory-like manner, but 3D effects are captured through an iterative procedure until all sections are operating at their corresponding viscous $\left(C_{l}, \alpha\right)$ point.

The aerodynamic model coupled with the aircraft equations of motion results in a low-order, medium-fidelity framework for flight dynamics simulation. Two different test cases have been studied to exhibit its capabilities and demonstrate the impact of unsteady and post-stall aerodynamics on aircraft behavior. First, the effect of neglecting unsteady effects has been shown on the flight-dynamics modes of a HALE UAV configuration (for attached flow conditions). While quasi-steady approximations based on vortex lattice aerodynamics or classical simplifications of the longitudinal equations predict the phugoid mode remarkably well, the discrepancy is significant for the short period mode, even qualitatively. Next, the combined unsteady as well as post-stall aerodynamics has been exercised in an asymmetric maneuver of a representative of a light general aviation aircraft, predicting a spiral motion that follows an asymmetric stall in a very efficient manner.

The methodology presented exhibits the potential for real-time flight simulation, and hence could supersede the conventional look-up table approach in a wide range of scenarios. Ongoing work focuses on speeding up the numerical implementation and on incorporating structural dynamics to cater for aeroelastic effects. The resulting model is expected to provide a powerful toolbox for the simulation of both flexible and rigid aircraft, including aerodynamicsin-the-loop of rigid aircraft, near real-time simulation of stiffer vehicles with linear structural behavior, and fully nonlinear dynamics of very flexible UAVs. 


\section{Acknowledgments}

The first author was supported by a fellowship from the Department of Defense SMART Scholarship. The program is gratefully acknowledged.

This work represents a collaborative effort between North Carolina State University (Raleigh, NC, USA) and the University of Surrey (Guildford, Surrey, UK). Travel funds for the authors have been provided by the University Global Partnership Network. This program is gratefully acknowledged.

\section{References}

[1] Cook, M. V., Flight Dynamics Principles: A Linear Systems Approach to Aircraft Stability and Control, Elsevier Ltd, 2nd ed., 2007.

[2] Ghoreyshi, M., Badcock, K. J., and Woodgate, M. A., "Accelerating the Numerical Generation of Aerodynamic Models for Flight Simulation," Journal of Aircraft, Vol. 46, No. 3, May-June 2009, pp. 972-980.

[3] Da Ronch, A., Ghoreyshi, M., and Badcock, K. J., "On the generation of flight dynamics aerodynamic tables by computational fluid dynamics," Progress in Aerospace Sciences, Vol. 47, No. 8, November 2011, pp. 597-620.

[4] Katz, J. and Plotkin, A., Low-Speed Aerodynamics, Cambridge Aerospace Series, Cambridge University Press, 2 nd ed., 2001.

[5] Murua, J., Palacios, R., and Graham, J. M. R., "Applications of the Unsteady Vortex-Lattice Method in Aircraft Aeroelasticity and Flight Dynamics," Progress in Aerospace Sciences, Vol. 55, 2012, pp. 46-72.

[6] Mukherjee, R. and Gopalarathnam, A., "Poststall Prediction of Multiple-Lifting-Surface Configurations Using a Decambering Approach,” Journal of Aircraft, Vol. 43, No. 3, May-June 2006, pp. 660-668.

[7] Gopalarathnam, A., Paul, R., and Petrilli, J., “Aerodynamic Modeling for Real-Time Flight Dynamics Simulation (Invited)," AIAA Paper 2013-0969, January 2013.

[8] Murua, J., Martínez, P., Climent, H., van Zyl, L. H., and Palacios, R., “T-tail flutter: potential-flow modelling and experimental validation,” Tech. rep., Bristol, UK, June 2013.

[9] Gopalarathnam, A. and Segawa, H., "Use of Lift Superposition for Improved Computational Efficiency of Wing Post-Stall Prediction,” AIAA Paper 2008-7049, 2008.

[10] Gopalarathnam, A. and Sutton, M., "A Rapid Aerodynamic Prediction Method for Simulation of Post-Stall Flight Dynamics," AIAA Paper 2010-8123, 2010.

[11] Gopalarathnam, A. and Paul, R., "Simulation of Flight Dynamics with an Improved Post-Stall Aerodynamics Model," Aiaa paper, 2012.

[12] Cho, J. and Cho, J., "Calculation of nonlinear aerodynamic characteristics of a wing using a 3-D panel method," International Journal for Numerical Methods in Fluids, Vol. 56, 2008, pp. 23-35.

[13] Tani, I., “A Simple Method of Calculating the Induced Velocity of a Monoplane Wing,” Rep. No. 111 (vol. 9, 3), Aero. Res. Inst., Tokyo Imperial Univ., August 1934.

[14] Sears, W. R., "Some Recent Developments in Airfoil Theory," Journal of The Aeronautical Sciences, Vol. 23, May 1956, pp. 490-499.

[15] Tseng, J. B. and Lan, C. E., "Calculation of Aerodynamic Characteristics of Airplane Configurations at High Angles of Attack," NASA CR 4182, 1988.

[16] Anderson, J. D., Corda, S., and VanWie, D. M., "Numerical Lifting Line Theory Applied to Drooped Leading-Edge Wings Below and Above Stall," Journal of Aircraft, Vol. 17, No. 12, 1980, pp. 898-904.

[17] van Dam, C. P., Kam, J. C. V., and Paris, J. K., "Design-Oriented High-Lift Methodology for General Aviation and Civil Transport Aircraft," Journal of Aircraft, Vol. 38, No. 6, November-December 2001, pp. 1076-1084.

[18] Selig, M., "Modeling Full-Envelope Aerodynamics of Small UAVs in Realtime,” AIAA Paper 2010-7635, 2010.

[19] Murua, J., Palacios, R., and Graham, J. M. R., "Assessment of Wake-Tail Interference Effects on the Dynamics of Flexible Aircraft," AIAA Journal, Vol. 50, No. 7, July 2012, pp. 1575-1585.

[20] Simpson, R., Palacios, R., and Murua, J., "Induced drag calculations in the unsteady vortex lattice method," AIAA Journal, 2013.

[21] Bunge, R. A. and Kroo, I. M., "Compact Formulation of Nonlinear Inviscid Aerodynamics for Fixed-Wing Aircraft," AIAA Paper 2012-2771, June 2012.

[22] Willis, D. J., Peraire, J., and White, J. K., "A Combined pFFT-Multipole Tree Code, Unsteady Panel Method with Vortex Particle Wakes," International Journal for Numerical Methods in Fluids, Vol. 53, 2007, pp. 1399-1422.

[23] Murua, J., Flexible-Aircraft Dynamics with a Geometricallly-Nonlinear Description of the Unsteady Aerodynamics, Ph.D. thesis, Imperial College London, 2012. 
[24] Paul, R. and Gopalarathnam, A., "Iteration schemes for rapid post-stall aerodynamic prediction of wings using a decambering approach (Submitted)," International Journal for Numerical Methods in Fluids.

[25] Klein, V. and Morelli, E. A., Aircraft System Identification Theory and Practice, American Institute of Aeronautics and Astronautics, 2006.

[26] Allerton, D., Principles of Flight Simulation, John Wiley and Sons Ltd., 2009.

[27] Patil, M. J., Hodges, D. H., and Cesnik, C. E. S., "Nonlinear Aeroelasticity and Flight Dynamics of High-Altitude LongEndurance Aircraft," Journal of Aircraft, Vol. 38, No. 1, 2001, pp. 88-94.

[28] Ashley, H., Engineering Analysis of Flight Vehicles, Addison-Wesley Aerospace Series, Addison-Wesley Publishing Co., 1974.

[29] Roskam, J., Airplane Flight Dynamics and Automatic Flight Controls, DAR Corporation, 2007.

[30] Petrilli, J., Paul, R., and Gopalarathnam, A., “A CFD Database for Airfoils and Wings at Post-Stall Angles of Attack,” AIAA Paper 2013-2916, June 2013.

[31] Drela, M., “XFOIL: An Analysis and Design System for Low Reynolds Number Airfoils," Low Reynolds Number Aerodynamics, edited by T. J. Mueller, Vol. 54 of Lecture Notes in Engineering, Springer-Verlag, New York, June 1989, pp. 1-12. 